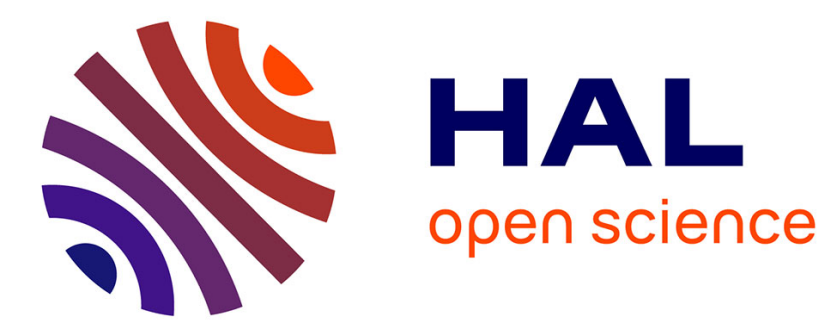

\title{
Modelling Organ Deformation Using Mass-Springs and Tensional Integrity
}

Pierre-Frédéric Villard, Wesley Bourne, Fernando Bello

\section{To cite this version:}

Pierre-Frédéric Villard, Wesley Bourne, Fernando Bello. Modelling Organ Deformation Using Mass-

Springs and Tensional Integrity. ISBMS, 2008, London, United Kingdom. pp.221-226. hal-00849197

\section{HAL Id: hal-00849197 \\ https://hal.inria.fr/hal-00849197}

Submitted on $30 \mathrm{Jul} 2013$

HAL is a multi-disciplinary open access archive for the deposit and dissemination of scientific research documents, whether they are published or not. The documents may come from teaching and research institutions in France or abroad, or from public or private research centers.
L'archive ouverte pluridisciplinaire HAL, est destinée au dépôt et à la diffusion de documents scientifiques de niveau recherche, publiés ou non, émanant des établissements d'enseignement et de recherche français ou étrangers, des laboratoires publics ou privés. 


\title{
Modelling Organ Deformation using Mass-springs and Tensional Integrity
}

\author{
Pierre-Frédéric Villard, Wesley Bourne, and Fernando Bello \\ Biosurgery and Surgical Technology Department, Imperial College London \\ 10th Floor QEQM, St Mary's Hospital \\ Praed Street, London W2 1NY, UK \\ $\{$ p.villard, f.bello\}@imperial.ac.uk
}

\begin{abstract}
In this paper we propose a new framework for deformation modelling based on a combined mass spring and tensional integrity method. The synergy between balanced tension and compression components offered by the tensegrity model helps the deforming organ retain its shape more consistently. We chose the diaphragm as our test object given its heterogeneous composition (muscle and tendon) and its importance in radiotherapy and other interventional procedures. Diaphragm motion can significantly influence surrounding organs such as lungs and liver. Our system permits the control of simulated diaphragm motion and deformation by at least two independent parameters.
\end{abstract}

Key words: Deformation model, mass-spring, tensegrity, interventional radiology

\section{Introduction}

Our work focuses on the modelling of the intrinsic deformation and motion of organs. Such modelling is highly relevant in the planning and execution of treatments and interventions, as well as for simulation used in the context of training and procedure rehearsal. The vast number of techniques used for organ deformation modelling can be classified into two different categories: the heuristic models such as deformable spline or mass spring, and Continuum-mechanical approach such as the FEM [1]. In the context of procedure training, rehearsal or guidance, real time is vital, therefore we explicitly chose a mass-spring system as it allows reasonably realistic behaviour while supporting near real time performance.

On the whole, organs have an heterogenous composition that can be expected to influence their behaviour and response to external stimuli. In order to incorporate this heterogeneity, we added the tensional integrity formulation (tensegrity) [2] to a mass-spring system to rigidify relevant tissue areas and result in a more realistic behaviour. The diaphragm example presented here illustrates a typical heterogeneity where the muscle tissue can be modelled by a mass-spring system and the tendinous part with tensegrity. 


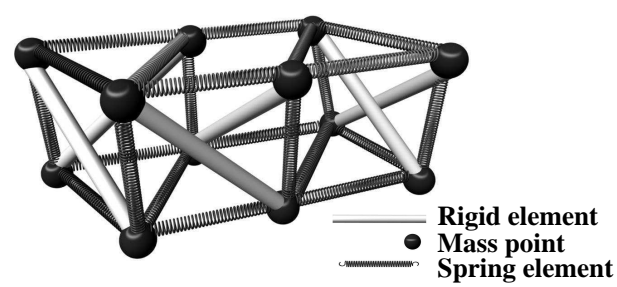

Fig. 1. A tensegrity element (12 mass points, 6 rigid elements and 22 springs)

\section{Method}

\subsection{Tensegrity definition}

The word "tensegrity" comes from a contraction of "Tensional integrity". It is used to define a mechanical system with components that combine tension and compression in such a way as to enable the whole system to receive and apply forces, tensions and pressures. Tension is continuous and compression discontinuous, such that continuous pull is balanced by equivalently discontinuous pushing forces.

Tensegrity systems are composed of two types of elements: the elastic elements, which give the system tension, and the rigid elements, which have a constant length and that will exert compression forces (Fig. 1). This concept of tensegrity is used in many areas. The term "tensegrity" was first explored by artist Kenneth Snelson to produce sculptures such as his 18 meter high Needle Tower in 1968. Imbert [2] states that a wide variety of natural systems, including carbon atoms, water molecules, proteins, viruses, cells, tissues and even humans and other living creatures, are constructed using tensegrity, a common form of architecture. Imbert's view of the cell as a tensegrity structure may help explain why cells in tissue culture spread out and flatten when grown on rigid glass of plastic petri dishes but, when on a flexible surface, the cells contract and become spherical. Therefore, tensegrity is a formulation that seems well suited to organ deformation modelling. The next section explains how tensegrity has been incorporated into our simulation framework.

\subsection{Implementation}

Our solution makes use of the Java3D API (http://java.sun.com/products/javamedia/3D), which is available for all major platforms. We have defined classes to describe topologies, nodes, forces and other required notions to create physical simulations. Topologies can be imported via a mesh parser or can be manually created. Nodes are automatically created along with connections when the parser is used. We have achieved real-time performance by optimising data handling and parameter passing in such a way that the API is notified every time changes have been made to the underlying data. This allows the simulation to be 


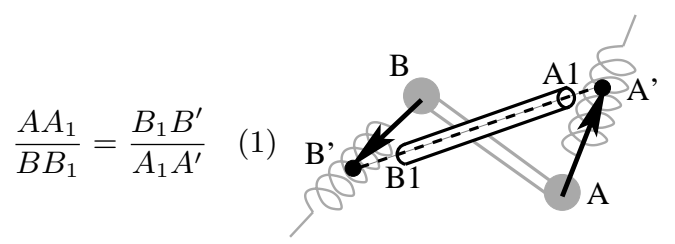

Fig. 2. Rigid links computation after spring influence

fully interactive and tuneable. The framework takes into account heterogeneity to combine object zones with only mass-spring and other zones with tensegrity. Objects are defined by two boundary surfaces. The outward appearance of volume within the two surfaces is created by an initial computation during runtime to create the tensile connections. The algorithm for the main simulation loop is as follows:

1. The elastic forces and the new positions of the nodes are computed as if there were no rigid links using the classical algorithm of the mass-spring method. The node displacements are computed at each time step by a Range-Kutta 2nd order approximation of the fundamental law of the dynamics.

2 . The rigid constrains are applied to ensure that the distance between two nodes remains constant as in [3]. Given two nodes $A$ and $B$ linked by a rigid link, $A^{\prime}$ and $B^{\prime}$ are their respective positions after applying the classical massspring algorithm (as if there was no rigid link). The real positions $A_{1}$ and $B_{1}$ considering the rigid links are given by Eq. (1) such that $A_{1}$ and $B_{1}$ remains on the line $A^{\prime} B^{\prime}$ (See Fig. 2).

3 . The new velocity is computed at each node.

\section{Results}

Soft tissue deformation and organ modelling are highly complex endeauvors. While a large number of modelling techniques have been proposed with varying degrees of accuracy, the behaviour of certain organs or structures may require the combination of characteristics offered by more than one technique. We propose such hybrid model incorporating the properties of classical mass-spring systems and tensegrity. To evaluate the performance of our hybrid model, we compared its behaviour to that of a simple mass-spring system and used our simulation framework to model and fine-tune diaphragm motion.

\subsection{Behaviour of tensegrity system (TS) and comparison to mass-spring system (MSS)}

We used a simple object (cube) to study and compare the behaviour of both systems. The simulations in Fig.3 show the influence of tensegrity. The objects are identical apart from the presence of rigid links. A sinusoidal force is applied to two of the upper nodes in identical directions. 

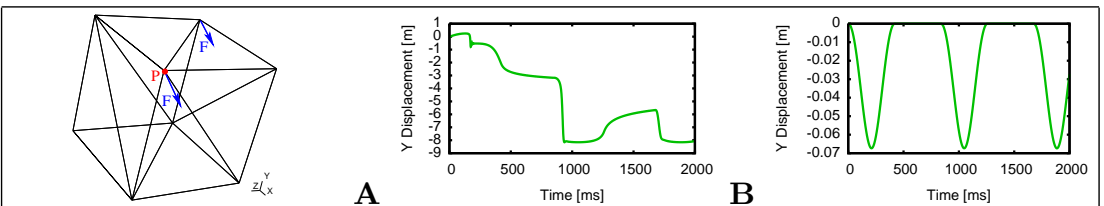

A force is applied to two nodes: positive along the $X$ axis, negative along the $Y$ axis and has an intensity given by a sinusoid curve $\mathrm{Y}$ displacement for point $\mathrm{P}$ on $\mathbf{A}$ ) MSS and $\mathbf{B}$ ) TS

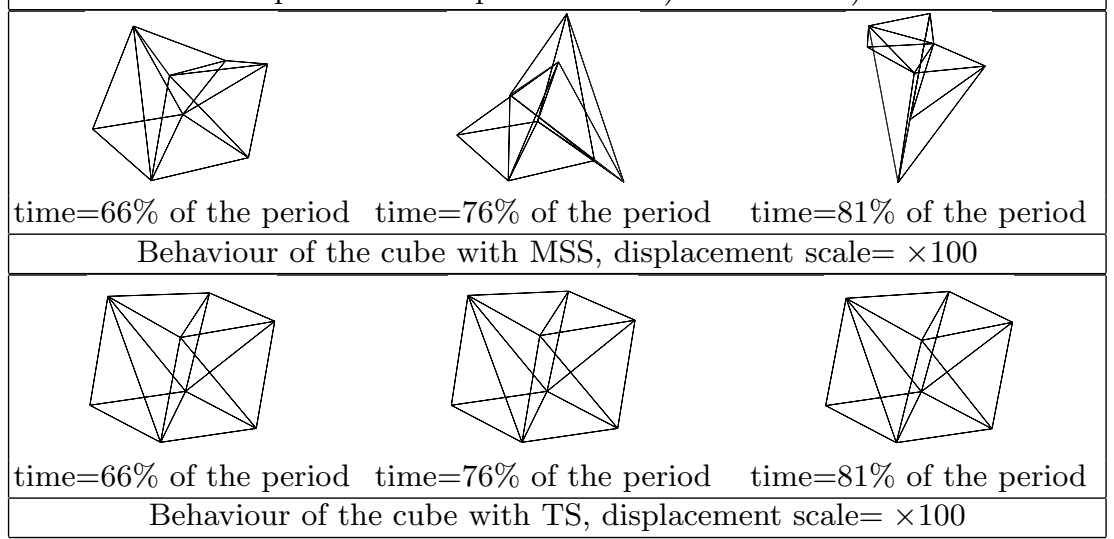

Fig. 3. Cube $\left(10 \times 10 \times 10 \mathrm{~m}^{3}\right)$ evolution with time. Top: boundary condition definitions, middle: results with MSS, bottom results with TS

Plotting the displacement for point $\mathrm{P}$ in both cubes (Fig. 3.A) further illustrates that the tensegrity system more accurately follows the input stimulus and is able to handle larger deformations without degradation (maximum Y displacement of $-0.075 \mathrm{~m}$ for the TS and of $-8 \mathrm{~m}$ for the MSS on Fig. 3.B).

\subsection{Application to the diaphragm}

Consistent behaviour and ability to withstand large forces are important attributes of our hybrid model. We used these characteristics to model diaphragm behaviour. As mentioned before, the diaphragm is an heterogeneous structure formed of muscular and tendinous tissue, thus ideally suited for our hybrid model. In the case of real diaphragms extracted from manually segmented patients, meshes are created using CGAL (www.cgal.org) library.

A simplified model from a detailed commercial anatomy set (Anatomium 3D by CF Lietzau 3D Special Service) was used to provide the anatomical parts shown in Fig. 4.left. Remeshing of the diaphragm was done to obtain a surface mesh composed of 11359 nodes and 22722 triangles. Tendinous tissue and muscle tissue were separated by a plane, and the rigid links of the tensegrity part were created by linking the upper surface to the lower surface with a heuristic algorithm (see Fig.4.right). 

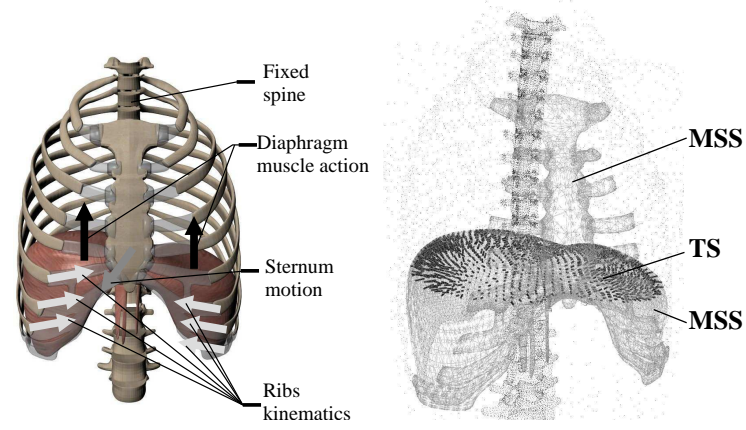

Fig. 4. Real physiological behaviour compared to simulation. Left: physiological motion of diaphragm with the influence of the spine, the sternum, the ribs and its intrinsic muscle contraction/relaxation. Right: wireframe representation of mass-spring elements and rigid elements.

The boundary conditions as well as the controlling forces are based on a physiological study. First, the anterior-posterior diameter increases because the sternum moves forwards as the ribs are raised. Secondly, the transverse diameter increases due to a "pump handle" movement which elevates the ribs. Both movements can be simulated by the combined motion of the ribs, the sternum and the cartilage. We first assume that the ribs are rigid and their motion can be modelled by a kinematics law based on the finite helical axis method as in [4]. The cartilage-sternum follows the motion of the ribs. This was modelled by a mass-spring system with a very low elasticity in order to ensure that it remains attached to the ribs while having a small internal deformation. The attachment points of the diaphragm are obtained such that the distance to the group ribs, sternum, cartilage is under a given threshold. Then, the vertical dimension increases due to diaphragm movement during inhalation. This motion can be simulated by the muscle action generation borrowed from Thalmann and Nedel [5]. We define and action line as a vertical line (inferior-superior) passing through given points and with a certain radius of action. The forces of contraction during inhalation or relaxation during exhalation are computed such that their intensity varies according to the distance from the action line. The displacement results of this simulation are shown in Fig. 5. To facilitate their visualisation, we display two slices in the anterior-posterior and the axial views.

\section{Discussion}

The simple cube experiment illustrates the ability of the tensegrity system to retain the shape of the object, even under considerably larger forces, while the mass-spring cube is crushed under much smaller forces. The tensegrity system more accurately follows the input stimulus and is able to handle larger deformations without degradation. 

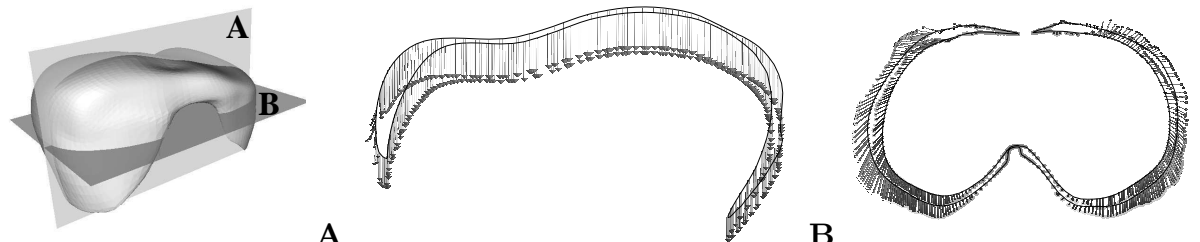

Fig. 5. Displacement vectors with scale 100 A: anterior posterior B: axial displacements

The diaphragm experiment illustrates the influence of the three phenomena mentioned above: the muscle relaxation that increases the height of the domes, the ribs rotation that presses the diaphragm on each side, and the sternum going backward that pushes the diaphragm. The physiological behaviour of this generic model was discussed with our clinical collaborator, who validated that the anterior-posterior, the transverse and vertical dimensions of the thorax are increased as illustrated by the model.

\section{Conclusion}

We have presented a model of heterogeneous organ deformation with tissues modelled using both mass-spring and tensegrity system. Comparing the behaviour between the two on a simple object showed the ability of the hybrid model to withstand larger forces and present a more consistent behaviour. Application of this method to diaphragm motion simulation has resulted in a powerful simulation framework that incorporates real boundary conditions, physiological constraints, and allows for the fine tuning of model parameters in real-time as the simulation is running. The different types of diaphragmatic motion are well noticed during the simulation and have been validated by our clinical collaborator. The main contribution of our work is a novel methodology to reproduce heterogeneity by combining elastic and tensile elements.

\section{References}

1. U.Meier, et.al: Real-time deformable models for surgery simulation: a survey. Comput Methods Programs Biomed. 77(3) (2005) 183-97

2. D.Ingber: Opposing views on tensegrity as a structural framework for understanding cell mechanics. J Appl Physiol 89 (2000) 1663-1678

3. A.Guillaume: 3d simulation of cell biomechanical behaviour. Master's thesis, University Claude Bernard Lyon 1, France (2004)

4. A.L.Didier, P.-F.Villard, J.Y.Bayle, M.Beuve, B.Shariat: Breathing Thorax Simulation based on Pleura Behaviour and Rib Kinematics. In IEEE, ed.: Information Visualisation - MediVis. (2007) 35-40

5. L.P.Nedel, D.Thalmann: Real time muscle deformations using mass-spring systems. In: CGI. (1998) 156-165 